\title{
A NOTE ON THE QUENCHING RATE
}

\author{
MAREK FILA AND JOSEPHUS HULSHOF
}

(Communicated by Barbara L. Keyfitz)

\begin{abstract}
We examine the quenching rate near a quenching point of a solution of a semilinear heat equation with singular powerlike absorption. A selfcontained result on similarity profiles allows us to improve a previous quenching theorem by Guo.
\end{abstract}

\section{INTRODUCTION}

Consider the problem

$$
\begin{cases}u_{t}-u_{x x}=-u^{-\beta}, & |x|<L, t>0 \\ u( \pm L, t)=1, & t>0 \\ u(x, 0)=u_{0}(x)>0, & |x| \leq L\end{cases}
$$

where $\beta \geq 1$ and $u_{0}( \pm L)=1$. The study of problems of this type was initiated by Kawarada in [K], and it is well known that there are data $u_{0}, L$ for which the solutions reach zero in finite time. We call this phenomenon quenching, and we say that $x_{0}$ is a quenching point for $u$ if there exist $0<T<\infty$, a sequence $\left\{\left(x_{n}, t_{n}\right)\right\}$ with $x_{n} \rightarrow x_{0}, t_{n} \uparrow T$, such that $u\left(x_{n}, t_{n}\right) \rightarrow 0$ as $n \rightarrow \infty$.

The quenching rate (as $t \uparrow T$ ) of the solution $u$ of (1.1) near a quenching point $x_{0}$ was studied in [G1], and for higher-dimensional radial problems in [G2]. In [G1] Guo showed that if

$$
u_{0}^{\prime \prime}-u_{0}^{-\beta} \leq 0,
$$

and $x_{0}$ is an arbitrary quenching point, then

$$
\lim _{t \uparrow T} u(x, t)(T-t)^{-\frac{1}{\beta+1}}=(\beta+1)^{\frac{1}{\beta+1}}
$$

uniformly for $\left|x-x_{0}\right| \leq C(T-t)^{1 / 2}$ for any positive constant $C$, but only under the assumption that $\beta \geq 3$.

The aim of this paper is to improve the quenching rate result from [G1] by proving (1.3) for all $\beta \geq 1$ and $u_{0}$ satisfying (1.2). Thus we also cover Kawarada's original problem $\left(\beta=1, u_{0} \equiv 1\right)$.

Received by the editors November 15, 1989 and, in revised form, May 8, 1990.

1980 Mathematics Subject Classification (1985 Revision). Primary 35K60; Secondary 34C11.

Key words and phrases. Semilinear parabolic equation, quenching rate, similarity variables, second-order ordinary differential equation, convexity. 
As in [G1], we use the similarity variables

$$
y:=\left(x-x_{0}\right)(T-t)^{-\frac{1}{2}}, \quad s:=-\log (T-t), \quad w(y, s):=u(x, t)(T-t)^{-\frac{1}{\beta+1}}
$$

which lead to the equation

$$
w_{s}=w_{y y}-\frac{1}{2} y w_{y}-w^{-\beta}+\frac{1}{\beta+1} w
$$

in

$$
W:=\left\{(y, s):-\left(x_{0}+L\right) e^{\frac{1}{2} s}<y<\left(L-x_{0}\right) e^{\frac{1}{2} s}, s>-\log T\right\},
$$

with lateral Dirichlet boundary conditions given by

$$
w\left(-\left(x_{0}+L\right) e^{\frac{1}{2} s}, s\right)=w\left(\left(L-x_{0}\right) e^{\frac{1}{2} s}, s\right)=e^{\frac{1}{\beta+1} s}
$$

for $s>-\log T$. The method of [GK], which was modified for quenching problems in [G1] and [G2], yields (1.3) if we can show that the only possible candidate for a limit element of $w(y, s)$ (as $s \uparrow \infty$ ) is the constant solution of

$$
w_{y y}-\frac{1}{2} y w_{y}=w^{-\beta}-\frac{1}{\beta+1} w .
$$

We examine this last equation in $\S 2$. In $\S 3$ we apply the results of $\S 2$ to the quenching problem.

\section{THE SIMILARITY PROFILES}

In this section we consider positive global solutions of the following ordinary differential equation:

$$
w^{\prime \prime}(y)-\frac{y w^{\prime}(y)}{2}=f(w(y)):=w(y)^{-\beta}-\frac{w(y)}{\beta+1}
$$

where $y$ is a real variable, and $\beta \geq 1$ is a real parameter. By global we mean that $w(y)$ is defined for all $y$. Throughout this section all solutions are tacitly assumed to be global and positive. The result we need is the following theorem.

Theorem 2.1. Let $\beta \geq 1$. Then, as $y \downarrow-\infty$ and/or $y \uparrow+\infty$, every nonconstant solution of (2.1) is eventually strictly convex and tends to $+\infty$.

The proof of Theorem 2.1 will be a consequence of a number of lemmas stated and proved below.

Lemma 2.2. A solution of (2.1) cannot be nonincreasing near $y=+\infty$ unless it is identically equal to

$$
k=k(\beta):=(\beta+1)^{\frac{1}{\beta+1}},
$$

which is the unique positive zero of $f$.

Proof. We argue by contradiction and suppose that there exists a nonconstant solution $w$ which is decreasing for large positive values of $y$. It is easily seen 
from (2.1) that $w$ then has to drop below the value $k$ defined by (2.2). Using the variation of constants formula we obtain

$$
w^{\prime}(y)=e^{\frac{1}{4} y^{2}}\left(w^{\prime}(0)+\int_{0}^{y} e^{-\frac{1}{4} \eta^{2}} g(\eta) d \eta\right),
$$

where $g(y):=f(w(y))$. Since $w(y)$ decreases to a nonnegative limit as $y \uparrow \infty$, this can be rewritten as

$$
w^{\prime}(y)=-e^{\frac{1}{4} y^{2}} \int_{y}^{\infty} e^{-\frac{1}{4} \eta^{2}} g(\eta) d \eta .
$$

However, the function $g(y)$ is positive and bounded away from zero for sufficiently large $y$, and the integral

$$
\int_{0}^{\infty} e^{\frac{1}{4} y^{2}} \int_{y}^{\infty} e^{-\frac{1}{4} \eta^{2}} g(\eta) d \eta d y
$$

diverges to $\infty$. This together with (2.3) implies that $w(y)$ cannot remain positive as $y \uparrow \infty$, a contradiction. Q.E.D.

The following lemmas are inspired by [FFM].

Lemma 2.3. Let $w(y)$ be a solution of (2.1) and define the function $J(y)$ by

$$
J(y)=e^{-\frac{1}{4} y^{2}}\left(w^{\prime}(y) w^{\prime \prime}(y)+\frac{w(y) w^{\prime \prime \prime}(y)}{\beta}\right) .
$$

Then $J$ satisfies

$$
J^{\prime}(y)=e^{-\frac{1}{4} y^{2}} \frac{1}{2 \beta}\left((\beta+1) y w^{\prime}(y) w^{\prime \prime}(y)+(\beta-1) w^{\prime}(y)^{2}\right)
$$

Proof. As in [FFM] one differentiates (2.1) twice, multiplies by $w / \beta$, and adds the result to $(2.1)$ multiplied by $w^{\prime \prime}$. Eliminating $w^{\prime \prime \prime}$ using the first derivative of (2.1) and inserting the explicit form of $f$ the identity is obtained after multiplication by $e^{-\frac{1}{4} y^{2}}$. Q.E.D.

Lemma 2.4. Let $w(y)$ be a solution of (2.1) with $w^{\prime}(0)=0$ and $0<w(0)<k$. Then $w$ is a strictly convex function.

Proof. First we observe that $w^{\prime \prime}(y)>0$ for small values of $y$ and that $J(0)=$ 0 . By (2.4) $J^{\prime}(y)>0$ as long as $w^{\prime \prime}(y)>0$, and the same holds of course for $w^{\prime}(y)$ since $w^{\prime}(0)=0$. Now suppose that there is some $y_{0}>0$ such that $w^{\prime \prime}\left(y_{0}\right)=0$. We may assume that $y_{0}$ is the first value for which this happens. But then $w^{\prime \prime \prime}\left(y_{0}\right) \leq 0$, implying $J\left(y_{0}\right) \leq 0$. Contradiction. Q.E.D.

Lemma 2.5. Let $w(y)$ be a nonconstant solution of (2.1), which is decreasing on some subinterval of the positive reals. Then $w(y)$ attains a positive minimum in a unique positive $y_{1}$ and is strictly convex for $y \geq y_{1}$.

Proof. By Lemma 2.2 there has to be a minimal positive value $y_{1}$ such that $w^{\prime}\left(y_{1}\right)=0$ and $w^{\prime \prime}\left(y_{1}\right) \geq 0$. Since $w(y)$ is not a constant, it follows that $w^{\prime \prime}\left(y_{1}\right)>0$. Differentiating $(2.1)$ we see that consequently $w^{\prime \prime \prime} \cdot\left(y_{1}\right)>0$, so 
that $J\left(y_{1}\right)>0$. The remainder of the proof is identical to the proof of Lemma 2.4 and therefore left to the reader. Q.E.D.

Proof of Theorem 2.1. Suppose that $w(y)$ is a nonconstant solution of (2.1), for which the statement is false. By Lemma 2.5, it has to be nondecreasing on the positive reals, and by symmetry, it has to be nonincreasing on the negative reals. Thus $w^{\prime}(0)=0$, and obviously $0<w(0)<k$. But then we can apply Lemma 2.4 and arrive at a contradiction again. Q.E.D.

Next we state two corollaries, which will be applied in $\S 3$.

Corollary 2.6. Let $\beta>1$ and let $w(y)$ be a nonconstant solution of (2.1). Then $w^{\prime \prime \prime}(y)$ is positive near $y=+\infty$ and/or negative near $y=-\infty$.

Proof. This follows immediately from Theorem 2.1 and differentiation of (2.1), because $\frac{1}{2}+f^{\prime}(w)$ tends to a positive constant as $w \rightarrow+\infty$. Q.E.D.

Corollary 2.7. Let $\beta=1$ and let $w(y)$ be a nonconstant solution of (2.1). Then $w(y) w^{\prime \prime}(y) \rightarrow+\infty$ as $y \downarrow-\infty$ and/or $y \uparrow+\infty$.

Proof. It follows from the proofs of Lemmas 2.4 and 2.5 that $J(y)$ is positive and bounded away from zero near $y=+\infty$ and/or near $y=-\infty$. Since $\beta=1$, we have that

$$
\left(w(y) w^{\prime \prime}(y)\right)^{\prime}=J(y) e^{\frac{1}{4} y^{2}} \rightarrow+\infty,
$$

as $y \downarrow-\infty$ and/or $y \uparrow+\infty$. Hence the corollary follows. Q.E.D.

\section{THE QUENCHING RATE}

Our main result is the following theorem.

Theorem 3.1. Let $\beta \geq 1$. Assume that $u$ is a solution of (1.1) which quenches in a finite time $T$, and assume that $u_{0}$ satisfies (1.2). Let $x_{0}$ be an arbitrary quenching point. Then

$$
\lim _{t \uparrow T} u(x, t)(T-t)^{-\frac{1}{\beta+1}}=k(\beta)=(\beta+1)^{\frac{1}{\beta+1}}
$$

uniformly for $\left|x-x_{0}\right| \leq C(T-t)^{\frac{1}{2}}$ for any positive constant $C$.

Proof. In the case $\beta>1$, it is known (see $[\mathrm{Gl},(3.10)]$ ) that

$$
c_{1} \leq w(y, s) \leq c_{2}|y|+c_{3}
$$

for some positive constants $c_{1}, c_{2}, c_{3}$, and any $(y, s) \in W$. Since (1.5) and (1.6) admit an energy functional, $w(y, s)$ tends (as $s \uparrow \infty$ ) to a positive function $w_{\infty}(y)$, which satisfies $(2.1)$ for all real $y$ (see $\S 3$ of [G1] for more details). Combining (3.1) with Theorem 2.1 and Corollary 2.6, we conclude that $w_{\infty} \equiv k(\beta)$.

For $\beta=1$ we still have, by the same reasoning as in [G1, Lemma 3.1], that

$$
c_{1} \leq w(y, s)
$$


for some positive constant $c_{1}$ and any $(y, s) \in W$. By the maximum principle, (1.2) implies that $u_{t} \leq 0$, and hence $u u_{x x} \leq 1$ for $|x| \leq L$ and $0<t<T$. Using (1.4) this yields

$$
w w_{y y} \leq 1
$$

in $W$. By (3.2), therefore, the growth of $w$ in $y$ is at most quadratic, which allows us again to proceed along the lines of $\S 3$ in [G1]. Thus here too we have that $w(y, s)$ tends to a positive function $w_{\infty}(y)$, which satisfies $(2.1)$ for all real $y$. From Corollary 2.7 and (3.3), it follows that $w_{\infty} \equiv k(\beta)$. Q.E.D.

\section{ACKNOWLEDGMENTS}

This paper was written during a visit of the first author to the University of Leiden financed by an E.E.C. grant. Both authors wish to thank L. A. Peletier for a number of fruitful discussions.

\section{REFERENCES}

[FFM] A. Friedman, J. Friedman, and B. McLeod, Concavity of solutions of nonlinear ordinary differential equations, J. Math. Anal. Appl. 131 (1988), 486-500.

[GK] Y. Giga and R. V. Kohn, Asymptotically self-similar blow-up for semilinear heat equations, Comm. Pure Appl. Math. 38 (1985), 297-319.

[G1] J. Guo, On the quenching behavior of the solution of a semilinear parabolic equations, IMA preprint 447, 1988.

[G2] - On the semilinear elliptic equation $\Delta w-\frac{1}{2} y \nabla w+\lambda w-w^{-\beta}=0$ in $\mathbf{R}^{n}$, unpublished.

[K] H. Kawarada, On solutions of initial boundary value problem for $u_{t}=u_{x x}+\frac{1}{1-u}$, Publ. Res. Inst. Math. Sci. 10 (1975), 729-736.

Department of Mathematical Analysis, Comenius University, Mlynská dolina, 84215 Bratislava, Czechoslovakia

Mathematical Institute, Leiden University, P.O. Box 9512, 2300 RA Leiden, The NETHERLANDS 